\title{
Nonlinear Sinusoidal Waves and Their Superposition in Anharmonic Lattices
}

\author{
Yuriy A. Kosevich \\ Department of Physics, Bilkent University, 06533 Bilkent, Ankara, Turkey \\ and National Surface and Vacuum Research Centre, 117334 Moscow, Russia
}

(Received 16 June 1993)

\begin{abstract}
A new type of finite-amplitude traveling or standing wave with an exact sinusoidal form and a short commensurate wavelength is predicted to exist in lattices with cubic and/or quartic anharmonic potential between any arbitrary number of nearest and non-nearest neighbors. Fast traveling nonlinear sinusoidal waves (NSW) can generate sinusoidal lattice solitons. Superposition of two NSW or sinusoidal solitons propagating in opposite directions can result in the formation of an extended or a localized standingwave eigenmode. New exact solutions for localized standing-wave structures are found within a rigorous discrete-lattice approach.
\end{abstract}

PACS numbers: $63.70 .+\mathrm{h}, 63.20 . \mathrm{Pw}, 63.20 . \mathrm{Ry}$

The only known exact solution of nonlinear discretelattice classical potential equations of motion concerns the case of the lattice with exponential nearest-neighbor anharmonic potential (Toda lattice) [1]. The exact periodic solution in the Toda lattice has the form of a cnoidal wave with arbitrary wavelength, which transforms into a sinusoidal wave only in the small-amplitude limit. Sinusoidal waves, being the fundamental low-lying excitations (phonon eigenmodes) of the lattice, are particularly important in its dynamics. In this Letter we present an exact solution of nonlinear discrete-lattice equations of motion of the one-dimensional (1D) anharmonic lattice in the form of finite-amplitude traveling or standing sinusoidal waves with a short and commensurate wavelength. The lattice is characterized by harmonic and cubic and/or quartic anharmonic interparticle potential. The case of the lattice with nearest-neighbor harmonic and cubic or quartic anharmonic potential corresponds to the famous Fermi-Pasta-Ulam model, for which the recurrence phenomenon in the nonlinear motion was revealed for the first time in computer simulations [2]. Because of the commensurability of the characteristic wavelength with the lattice period, nonlinear sinusoidal waves (NSW) are exact eigenmodes of a 1D lattice with cubic and/or quartic anharmonic potential between any arbitrary number of nearest and non-nearest neighbors. In anharmonic lattices of higher dimensionality, plane NSW can propagate in certain directions (of high symmetry). Contrary to the linear sinusoidal waves, the frequency of NSW depends on the amplitude, while the wave number is determined (fixed) by the anharmonic interactions. Therefore large-amplitude vibrational eigenstates of the anharmonic lattice can be classified with the help of NSW with amplitude-dependent frequency (and velocity) and a given wavelength, and hence NSW can contribute to the specific heat and energy transport in the system. NSW with velocity larger than the harmonic wave velocity can generate sinusoidal lattice solitons of substantially different form than that of solitons of the Korteweg-de Vries (KdV) (or modified $\mathrm{KdV}$ equation), which describes the weakly nonlinear long-wavelength dynamics of the lattice. Strongly localized solitary "peaks" propaga- ting at speeds larger than the harmonic wave speed were revealed in computer simulations of anharmonic vibrations of one- and two-dimensional lattices [3], while recent analytical and numerical studies [4,5] concern only the discrete-lattice solitons moving with speeds much lower than the harmonic wave speed. Superposition of two NSW propagating in opposite directions with equal amplitudes can result in the formation of a stable standing-wave extended eigenmode, while superposition (during resonant head-on collision) of two sinusoidal solitary waves can result in the formation of a localized nonlinear model. Such a phenomenon, which is quite unusual for the behavior of solitary waves in completely integrable systems where solitons restore their coherent shapes after colliding [6,7], was also revealed in computer simulations [3]. New exact solutions for strongly localized dark-profile standing-wave lattice structures are found. We claim that the existence of NSW in anharmonic lattices is not occasional since localized shortwavelength lattice vibrational modes with sinusoidal envelope and small amplitude-independent spatial width are exact solutions of the continuous envelope-function equation with the nonlinear gradient terms [8]. Solitary waves with compact support (compactons) and sinusoidal profile were recently obtained as the solutions of the continuous KdV-like equations with nonlinear dispersion [9]. [Continuous $\mathrm{KdV}$-like equations with nonlinear dispersion, which represent the corresponding long-wavelength limit of the below discrete-lattice equations (2), also have exact solutions in the form of NSW [10]].

We start from the model of a monatomic periodic chain with anharmonic potential of the order $r=3$ and/or $r=4$ between $\delta \geq 1$ nearest and non-nearest neighbors,

$$
U=\sum_{n} \sum_{\gamma=2}^{r} \sum_{\delta=1} \frac{1}{\gamma} K_{\gamma, \delta}\left(u_{n+\delta}-u_{n}\right)^{\gamma},
$$

where $u_{n}$ is the (real scalar) displacement of the $n$th particle from its equilibrium position and $K_{\gamma, \delta}$ are harmonic $(\gamma=2)$ and anharmonic force constants. The lowest order potential describing the anharmonicity of the longitudinal or pure transverse motion in a centrosymmetric $1 \mathrm{D}$ lattice corresponds to $r=3$ or $r=4$, respectively. From 
Eq. (1) we obtain the nonlinear discrete-lattice equations of motion:

$m \ddot{u}_{n}=\sum_{\gamma=2}^{r} \sum_{\delta=1} K_{\gamma, \delta}\left[\left(u_{n+\delta}-u_{n}\right)^{\gamma-1}-\left(u_{n}-u_{n-\delta}\right)^{\gamma-1}\right]$.

We are looking for the solution of Eqs. (2) for the displacements $u_{n}$ (or relative displacements $r_{n} \equiv u_{n+1}-u_{n}$ ) in the form of traveling (shifted) sinusoidal waves:

$$
\left(\begin{array}{l}
u_{n} \\
r_{n}
\end{array}\right)=A \cos (k n a-\omega t+\phi)+B
$$

In the lattice with quartic anharmonic nearestneighbor interaction (ANNI), NSW with $u_{n}$ pattern (3) are exact solutions of Eqs. (2) with the amplitudedependent frequency $\omega$ and definite wave number $k$ :

$$
\begin{aligned}
& m \omega^{2}=3 K_{2}+(27 / 4) K_{4} A^{2}, \\
& K_{4} A^{2} \sin ^{4}\left(\frac{1}{2} k a\right)\left[\sin ^{2}\left(\frac{1}{2} k a\right)-3 \cos ^{2}\left(\frac{1}{2} k a\right)\right]=0,
\end{aligned}
$$

where $K_{\gamma} \equiv K_{\gamma, 1}$. Equation (5) is obtained from requiring the absence of third (and correspondingly higher) harmonic contribution to NSW [3]. In the reducedBrillouin-zone picture, we find the "allowed" wave numbers: $k a= \pm \frac{2}{3} \pi$. In a $1 \mathrm{D}$ lattice with harmonic and quartic anharmonic interaction between $\delta \geq 2$ nonnearest neighbors, parameter $k a$ in Eq. (5) should be replaced by the parameter $k a \delta$, and the corresponding equation is also satisfied by the above values of the wave number $k$. Therefore NSW (3) with a commensurate wavelength $\lambda=3 a$ are exact eigenmodes of $1 \mathrm{D}$ lattice (1) with harmonic and quartic anharmonic interaction between any arbitrary number of nearest and non-nearest $(\delta \geq 1)$ neighbors. For wavelengths different from the above one, the term proportional to the left-hand side of Eq. (5) determines the generation of the higher harmonics of NSW (3). Therefore the generation is strongly suppressed for NSW with wave numbers close to the allowed ones.

NSW with $u_{n}$ pattern (3), edge Brillouin-zone wavelength $k a= \pm \pi$, and (amplitude-independent) upper cutoff frequency of harmonic oscillations $\left(m \omega^{2}=4 K_{2}\right.$ for $\delta=1$ ) are exact solutions of Eqs. (2) in a 1D lattice with cubic anharmonic interaction between any arbitrary number of nearest and non-nearest neighbors.

NSW with $r_{n}$ pattern (3) and frequency

$$
\begin{aligned}
m \omega^{2}= & 4 \sin ^{2}(k a / 2) \\
& \times\left\{K_{2}+2 K_{3} B+K_{4}\left[3 B^{2}+A^{2}\left(1+\frac{1}{2} \cos (k a)\right)\right]\right\}
\end{aligned}
$$

are exact eigenmodes of Eqs. (2) for $k a= \pm \pi$ and arbitrary $B$ in the chain with cubic ANNI $\left(K_{4}=0\right)$, and for $k a= \pm \frac{2}{3} \pi, \quad B=-K_{3} / 3 K_{4}$ in the chain with cubic +quartic ANNI (or with quartic ANNI, $K_{3}=0, B=0$ ).
Using trigonometric identities, it can be easily shown that linear superposition of two NSW with $u_{n}$ patterns (3) propagating in the same direction with $B=0$, equal amplitudes $A$ and different initial phases $\phi_{1,2}$ is also an exact eigenmode with the same amplitude $A$, if the phase difference $\phi_{1}-\phi_{2}$ is equal to $2 \pi\left(n \pm \frac{1}{3} \pi\right)$ with an integer $n$; i.e., if it coincides with one of the allowed values of $k a$ given by Eq. (5). Then, using the identity $u_{n+\delta}-u_{n}$ $=\sum_{\gamma}^{\delta=0}=1 r_{n+\gamma}$ and the above property of superposition of two NSW, it can be shown that NSW with $r_{n}$ pattern (3) are exact eigenmodes of Eqs. (2) with $|k a|=\frac{2}{3} \pi$ (or $|k a|=\pi$ ) in the lattice with cubictquartic (or cubic) anharmonic interaction between any arbitrary number $\delta \geq 1$ of nearest and non-nearest neighbors.

We can consider a "small-amplitude modulation" of NSW (3) in the form

$$
\begin{aligned}
\left(\begin{array}{l}
u_{n} \\
r_{n}
\end{array}\right)= & A \cos (k n a-\omega t+\phi) \\
& +\sum_{i} b_{i} \cos \left(k_{i} n a-\omega_{i} t+\phi_{i}\right)+B,
\end{aligned}
$$

where $b_{i} \ll A, k_{i}$, and $\omega_{i}$ are (small) amplitudes, wave numbers, and frequencies of modulation. Then from Eqs. (2) we can find that "linear" sinusoidal waves (7) (with amplitudes $b_{i}$ ) are lattice modes with the Goldstone harmonic-wave dispersion relation with the force constant renormalized by NSW (3). For instance, in the lattice with quartic ANNI, the $i$ th linear sinusoidal wave with $u_{n}$ pattern (7) and $k_{a}=\frac{2}{3} \pi$ has spectrum $m \omega_{i}^{2}$ $=4 \sin ^{2}\left(\frac{1}{2} k_{i} a\right)\left[K_{2}+(9 / 4) K_{4} A^{2}\right]$. In the lattice with cubic+quartic ANNI, the $i$ th linear sinusoidal wave with $r_{n}$ pattern (7) has a spectrum of the form similar to Eq. (6) (and with the same notation):

$$
\begin{aligned}
m \omega_{i}^{2}=4 \sin ^{2}\left(\frac{1}{2} k_{i} a\right)\{ & K_{2}+2 K_{3} B \\
& \left.+K_{4}\left[3 B^{2}+A^{2}\left(1+\frac{1}{2} \cos (k a)\right)\right]\right\} .
\end{aligned}
$$

Therefore finite-amplitude NSW (3), (4), and (6) with $\omega^{2}>0$ are stable eigenmodes of an anharmonic chain (1). Moreover, they represent a new basis for the classification of the vibrational eigenstates of the system.

In the lattice solitary wave, relative displacements of neighboring particles $r_{n}$ (as well as displacements $u_{n}$ or values $\Delta u_{n} \equiv u_{\infty}-u_{n}$, where $u_{\infty}$ is the finite displacement at infinity; see below) vanish at both infinities according to the exponential law, the inverse decay length $\kappa$ in which is determined by the linear part of Eqs. (2):

$$
\begin{aligned}
& r_{n} \propto \exp [ \pm \kappa(n a-c t)], \\
& 4 K_{2} \sinh ^{2}(\kappa a / 2)=m c^{2} \kappa^{2},
\end{aligned}
$$

where $c$ is a velocity of the lattice solitary wave. Equation (9) has a solution for the real $\kappa$ only in the case when velocity $c$ is larger than the harmonic wave velocity: $c>\sqrt{K_{2} a^{2} / m}$. So far as the (phase) velocity $c \equiv \omega / k$ of NSW (3) depends, in the general case, on the amplitude 
$A$ (or $B$ ), the form of the large-amplitude lattice solitons can be obtained by matching the sinusoidal $r_{n}$ pattern (3) of "supersonic" NSW (near corresponding zeros) with exponential tails (8). The matching can be performed by solving Eq. (2) for the particles (with $u_{n} \ll A$ or $\Delta u_{n} \ll A$ ) at the moving "border points" between the sinusoidal $r_{n}$ pattern (3) and (small-amplitude) tails (8). For this construction it is essential that fast lattice solitary waves (with $m c^{2} \gg K_{2} a^{2}$ ) have very short exponential tails: $\kappa a \gg 1$ [see Eq. (9)]. Therefore large-amplitude solitary waves in the anharmonic lattice (1) actually have sinusoidal form.

In the lattice with hard quartic ANNI $\left(K_{4}>0\right)$, any half period (between two successive zeros) of the cosine $r_{n}$ pattern (3) with $k a=\frac{2}{3} \pi, m \omega^{2}=3 K_{2}+\frac{9}{4} K_{4} A^{2}$, and $B=0$ corresponds to a half period (between zero and successive maximum) of the cosine $u_{n}$ pattern (3) with the amplitudes $A^{\prime}=A / \sqrt{3}$ and $\left|B^{\prime}\right| \leq A^{\prime}$. Thus a lattice solitary wave, which is described by a half period (or by a period) of the cosine $r_{n}$ pattern (3) with $B=0,-\pi / 2$ $<k n a-\omega t+\phi<+\pi / 2$ (or $-\pi / 2<k n a-\omega t+\phi<3 \pi$ ) 2 ), corresponds, e.g., to a steplike with $u-\infty=0$ and $u_{+\infty}=2 A / \sqrt{3}$ (or a pulselike with $u_{-\infty}=u+\infty=0$ ) cosine $u_{n}$ pattern (3): $u_{n}=u+\infty \cos ^{2}\left[\left(k n a-\omega t+\phi^{\prime}\right) / 2\right],-\pi$ $<k n a-k c t+\phi^{\prime}<0 \quad$ (or $\quad-\pi<k n a-\omega t+\phi^{\prime}<+\pi$ ). The former (steplike) solitary wave represents the generalization of the soliton of the (weakly nonlinear) modified $\mathrm{KdV}$ equation in the limit of large amplitudes, when $m c^{2} \gg K_{2} A^{2}$ and the soliton has small amplitudeindependent spatial width $\Delta=3 a / 2$. The latter (pulselike) lattice solitary wave has no counterpart in the weakly nonlinear limit. These most strongly localized fast solitary waves can be generalized to the traveling localized modes with three and more half periods of the cosine $r_{n}$ pattern (3).

In the lattice with cubic ANNI, large-amplitude solitary waves can be obtained by matching the cosine $r_{n}$ pattern (3) of supersonic NSW (with $k a=\pi$, $m \omega^{2}=4 K_{2}+8 K_{3} B, K_{3} B \gg K_{2}$ and $\left.A \geq|B|\right)$ with short exponential tails (8). Then the one-pulse $r_{n}$ pattern $\left[r_{n}=A \cos ^{2}[(k n a-k c t+\phi) / 2], \quad-\pi<k n a-k c t+\phi<\pi\right.$, $B=A$ ] corresponds to a one step with $u_{-\infty}=0$ and $u+\infty=A$ cosine $u_{n}$ pattern: $u_{n}=A \cos ^{2}[(k n a-k c t$ $\left.+\phi^{\prime}\right) / 2$ ], $-\pi<k n a-k c t+\phi^{\prime}<0$. This solitary wave represents the generalization of the soliton of the $\mathrm{KdV}$ equation in the large-amplitude limit, when $m c^{2} \gg K_{2} a^{2}$ and a steplike distribution of the lattice displacements $u_{n}$ is located on an interparticle spacing $a$. The two-pulse $r_{n}$ pattern corresponds to a two-step $u_{n}$ distribution (between $u-\infty=0$ and $u+\infty=2 A$ ), etc. In the lattice with cubicthard quartic ANNI, large-amplitude solitary waves with similar $u_{n}$ patterns can be obtained by matching the cosine $r_{n}$ pattern (3) of supersonic NSW [with $k a=\frac{2}{3} \pi, \quad m \omega^{2}=3 K_{2}+(9 / 4) K_{4} A^{2}-K_{3}^{2} / K_{4}, \quad B=-K_{3} /$ $3 K_{4}$ and arbitrary amplitude $\left.A \geq|B|\right]$ with short exponential tails (8).
We can consider a sinusoidal standing-wave mode as a superposition of two NSW (3) propagating in the opposite directions with equal amplitudes $A$ :

$$
\left(\begin{array}{l}
u_{n} \\
r_{n}
\end{array}\right)=\mathcal{A}(t) \cos (k n a+\alpha)+B \text {. }
$$

In the lattice with cubic anharmonicity, the mode with $u_{n}$ or $r_{n}$ pattern (10) is an exact solution of Eqs. (2) with $|k a|=\pi$, arbitrary $\alpha$, and $\mathcal{A}(t)=A \cos (\omega t)$ (where, e.g., $m \omega^{2}=4 K_{2}$ or $m \omega^{2}=4 K_{2}+8 K_{3} B$ in the lattice with the nearest-neighbor interaction). In the lattice with quartic (or cubic+quartic) anharmonicity, sinusoidal modes (10) are exact solutions of Eqs. (2) with $|k a|=\frac{2}{3} \pi$ and arbitrary $\alpha$, and also with $|k a|=\pi$ or $|k a|=\frac{1}{2} \pi$ and definite initial phase $\alpha$, which is determined from the requirement $\cos [3(k n a+\alpha)]= \pm \cos (k n a+\alpha) \quad($ e.g., $\quad \alpha=0)$. Time dependence of the amplitude $\mathcal{A}(t)$ of these modes is governed by the effective equation of motion of a decoupled (single) anharmonic oscillator. Thus we find the equations $m \ddot{\mathcal{A}}=-2 K_{2} \mathcal{A}-2 K_{4} \mathcal{A}^{3}, \quad m \ddot{\mathcal{A}}=-3 K_{2} \mathcal{A}$ $-(27 / 4) K_{4} \mathcal{A}^{3}$, and $m \ddot{\mathcal{A}}=-4 K_{2} \mathcal{A}-16 K_{4} \mathcal{A}^{3}$ for the modes with $u_{n}$ pattern (10) and $k a=\frac{1}{2} \pi, k a=\frac{2}{3} \pi$, and $k a=\pi$ in the lattice with quartic ANNI. For the mode with $r_{n}$ pattern (10) and $k a=\frac{2}{3} \pi, B=-K_{3} / 3 K_{4}$, we find the equation $m \ddot{\mathscr{A}}=-\left[3 K_{2}-\left(K_{3}^{2} / K_{4}\right)\right] \mathcal{A}-(9 / 4) K_{4} \mathcal{A}^{3}$ in the lattice with cubic and quartic ANNI (or quartic ANNI, $K_{3}=0, B=0$ ). From these equations we can exactly determine the period of oscillation $T$ of nonlinear sinusoidal mode (10) as a function of amplitude $\mathcal{A}_{\max }$ (or energy per single oscillator) (see, e.g., [11]). These equations can also be easily solved within a "rotating wave approximation" (RWA) when $\mathcal{A}(t)=A \cos (\omega t)$ and only a single frequency component is included in the time dependence. For the considered short-wavelength optical-like oscillations, this approximation holds due to the weakness of nonresonant interaction between the modes with fundamental frequency and its third harmonic.

In the lattice standing-wave localized mode with bright profile, the envelope of particle displacements $f_{n} \equiv(-1)^{n} u_{n}$ decays at both infinities according to the exponential law [cf. Eqs. (8) and (9)]:

$$
\begin{aligned}
& f_{n} \propto \exp ( \pm q a n) \cos (\omega t), \\
& 4 K_{2} \cosh ^{2}(q a / 2)=m \omega^{2} .
\end{aligned}
$$

Equation (12) has a solution for real $q$ only for the localized mode with frequency higher than the upper cutoff frequency of harmonic oscillations $\left(m \omega^{2}>4 K_{2}\right)$, and for the high frequency anharmonic mode the decay length of the exponential tail (11) is much shorter than interparticle spacing: $q a \gg 1$ for $m \omega^{2} \gg 4 K_{2}$. Therefore the $f_{n}$ pattern of the localized standing-wave mode can be obtained by matching (near corresponding minima) the envelope function $f_{n}=(-1)^{n} u_{n}$ of sinusoidal mode (10) (with oscillation period $T$ ) with short exponential tails (11) of the same (high) frequency $\omega=2 \pi / T$. In this way 
we can establish that in the lattice with hard quartic anharmonicity, two symmetric most strongly localized large-amplitude sinusoidal modes of odd and even parity exist, which correspond to a half period of the cosine envelope function $f_{n}=\mathcal{A}(t) \cos \left(\frac{1}{3} \pi n a+\alpha\right)$ with $\alpha=0$ or $\alpha=\frac{1}{6} \pi$ and have (approximate) displacement patterns $u_{n}=\mathcal{A}(t)\left(\ldots, 0,-\frac{1}{2}, 1,-\frac{1}{2}, 0, \ldots\right)$ or $u_{n}=\mathcal{A}(t)(\ldots, 0$, $-1,1,0, \ldots)$. Intrinsic localized modes with such displacement patterns were previously revealed in asymptotic analytical and numerical studies of the dynamics of monatomic lattice with hard quartic ANNI [12,13] (see also [8] for the continuous envelope-function description of these and other nonlinear localized modes). A slowly moving large-amplitude localized sinusoidal mode can also exist in the lattice, which has in the RWA the envelope $f_{n}=A \cos \left[k\left(n a-V_{g} t\right)\right] \cos (\Delta k-\omega t)$ [for $\mid k(n a$ $\left.\left.-V_{g} t\right) \mid \leq \frac{1}{2} \pi, \quad k a \simeq \frac{1}{3} \pi, \quad m \omega^{2} \simeq 3 K_{2}+(81 / 16) K_{4} A^{2}\right]$, where $V_{g}(\Delta k, A) \ll \sqrt{K_{2} a^{2} / m}$ and $\Delta k \ll k$ are the (small) group velocity and "reduced" wave number of the mode. In the lattice with cubic+hard quartic anharmonicity, a standing-wave localized mode with the one-period cosine $r_{n}$ pattern (10) exists [with $k a=\frac{2}{3} \pi, B=-K_{3}$ / $3 K_{4}, A \gg|B|$, and $m \omega^{2}=3 K_{2}+(27 / 16) K_{4} A^{2}-K_{3}^{2} / K_{4}$ in the RWA], which has (slightly) asymmetric $u_{n}$ and $f_{n}$ patterns. Essentially the formation of any of the above nonlinear sinusoidal standing-wave (or slowly moving) localized modes can occur in consequence of superposition (during resonant head-on collision) of two sinusoidal solitary waves [with the one-period cosine $r_{n}$ patterns (3)] propagating in opposite directions with equal (or close) amplitudes $A$, similar to the formation of the extended sinusoidal standing-wave mode (10).

Matching sinusoidal eigenmodes (10) with $k a=\frac{1}{2} \pi$, $k a=\frac{2}{3} \pi$, or $k a=\pi$, we can find new exact solutions for strongly localized transition regions between two extended standing-wave vibrational domains of definite wave number in the lattice with soft quartic ANNI $\left(K_{4}<0\right)$. Indeed, we reveal in the RWA that (at least) three extended eigenmodes [with the patterns $u_{n}=A_{0} \cos \left(\frac{1}{2} \pi n\right)$ $\times \cos \left(\omega_{0} t\right), u_{n}=A_{0}(\sqrt{4 / 3}) \cos \left(\frac{2}{3} \pi n+\frac{1}{6} \pi\right) \cos \left(\omega_{0} t\right)$, and $\left.u_{n}=A_{0} \cos (\pi n) \cos \left(\omega_{0} t\right)\right]$ exist in the lattice, in which particles oscillate with the same frequency $\omega_{0}$ $=\sqrt{12 K_{2} / 7 m}$ and amplitude $A_{0}=\sqrt{4 K_{2} / 21\left|K_{4}\right|}$. With the help of these eigenmodes, we easily obtain the exact form of (most strongly localized) domain walls between two wavelength-four modes $\left[u_{n}=A_{0}(\ldots,-1,0\right.$, $\left.1,0,-1,1,0,-1,0,1, \ldots) \cos \left(\omega_{0} t\right)\right]$, between two upper cutoff modes $\left[u_{n}=A_{0}(\ldots, 1,-1,1,0,-1,1,-1, \ldots)\right.$ $\times \cos \left(\omega_{0} t\right)$, between the upper cutoff and wavelengthfour modes $\left[u_{n}=A_{0}(\ldots,-1,1,-1,0,1,0,-1,0,1, \ldots)\right.$ $\left.\times \cos \left(\omega_{0} t\right)\right]$, etc. A link with the pattern $u_{n}$ $=A_{0}(0,-1,1,0) \cos \left(\omega_{0} t\right)$ can play the role of a kink in the upper cutoff (or wavelength-four) mode, while a link with the pattern $u_{n}=A_{0}(0,1,0) \cos \left(\omega_{0} t\right)$ can play the role of a kink in the $k a=\frac{2}{3} \pi$ (or upper cutoff) mode. All of these dark-profile localized structures in the lattice with interparticle anharmonic potential (1) substantially differ from similar structures in the lattice with on-site anharmonic potential, which have been recently observed in the lattice of coupled pendulums suspended in a gravitational field [14].

In conclusion, we have shown that finite-amplitude traveling or standing sinusoidal waves with a short and commensurate wavelength and, in general, amplitudedependent frequency, are exact eigenmodes of a $1 \mathrm{D}$ lattice with cubic and/or quartic anharmonic potential between any arbitrary number of nearest and non-nearest neighbors. New dark-profile localized standing-wave structures are predicted within a rigorous discrete-lattice approach. The existence of NSW influences the classification of the large-amplitude vibrational eigenstates, and therefore NSW must be considered in a complete thermodynamic description of the anharmonic lattices. Supersonic traveling NSW can generate sinusoidal lattice solitons and contribute to the energy transport in the system. The processes of the formation of standing-wave (or slowly moving) nonlinear sinusoidal modes, including localized ones, as a consequence of the superposition of two NSW traveling in opposite directions, can influence Anderson localization of vibrational states in disordered anharmonic solids.

I am grateful to M. J. Ablowitz for the useful discussion. I would like to thank Bilkent University for the hospitality and TUBITAK for the support of the work.

[1] M. Toda, in Theory of Nonlinear Lattices, edited by M. Toda, Solid State Sciences Vol. 20 (Springer, Berlin, $1981)$.

[2] E. Fermi, J. R. Pasta, and S. M. Ulam, in Collected Works of E. Fermi, edited by E. Segre (University of Chicago Press, Chicago, 1965).

[3] R. Bourbonnais and R. Maynard, Phys. Rev. Lett. 64, 1397 (1990)

[4] S. R. Bickham, A. J. Sievers, and S. Takeno, Phys. Rev. B 45, 10344 (1992).

[5] K. W. Sandusky, J. B. Page, and K. E. Schmidt, Phys. Rev. B 46, 6161 (1992).

[6] N. J. Zabusky and M. D. Kruskal, Phys. Rev. Lett. 15, 240 (1965)

[7] M. J. Ablowitz and H. Segur, Solitons and the Inverse Transform Method (SIAM, Philadelphia, 1981).

[8] Yu. A. Kosevich, Phys. Rev. B 47, 3138 (1993).

[9] P. Rosenau and J. M. Hyman, Phys. Rev. Lett. 70, 564 (1993).

[10] Yu. A. Kosevich (unpublished).

[11] L. D. Landau and E. M. Lifshitz, Mechanics (Pergamon, New York, 1976).

[12] A. J. Sievers and S. Takeno, Phys. Rev. Lett. 61, 970 (1988).

[13] J. B. Page, Phys. Rev. B 41, 7835 (1990).

[14] B. Denardo et al., Phys. Rev. Lett. 68, 1730 (1992). 